\title{
Cosmological simulations of low-mass galaxies: some potential issues
}

\author{
Pedro Colín ${ }^{1}$, Vladimir Avila-Reese ${ }^{2}$, and Octavio Valenzuela ${ }^{2}$ \\ ${ }^{1}$ Centro de Radioastronomía y Astrofísica, Universidad Nacional Autónoma de México, A.P. \\ 72-3 (Xangari), Morelia, Michoacán 58089, México \\ ${ }^{2}$ Instituto de Astronomía, Universidad Nacional Autónoma de México, A.P. 70-264, 04510, \\ México, D.F., México
}

\begin{abstract}
Cosmological Adaptive Mesh Refinement simulations are used to study the specific star formation rate $\left(\mathrm{sSFR}=\mathrm{SSF} / M_{s}\right)$ history and the stellar mass fraction, $f_{s}=M_{s} / M_{T}$, of small galaxies, total masses $M_{T}$ between few $\times 10^{10} \mathrm{M}_{\odot}$ to few $\times 10^{11} \mathrm{M}_{\odot}$. Our results are compared with recent observational inferences that show the so-called "downsizing in sSFR" phenomenon: the less massive the galaxy, the higher on average is its sSFR, a trend seen at least since $z \sim 1$. The simulations are not able to reproduce this phenomenon, in particular the high inferred values of sSFR, as well as the low values of $f_{s}$ constrained from observations. The effects of resolution and sub-grid physics on the SFR and $f_{s}$ of galaxies are discussed.
\end{abstract}

\section{Introduction}

Numerical N-body + hydrodynamical cosmological simulations have maturated enough as to predict some of the main properties of real galaxies; nevertheless, issues related to resolution and the so-called sub-grid physics are not settled yet. Therefore, inevitably the results of the simulations used here depends on the resolution and on the schemes and parameters used to model the physics of the unresolved scales. The current theoretical background of galaxy formation and evolution is the successful $\Lambda \mathrm{CDM}$ hierarchical clustering scenario. In this work, we simulate 'realistic' sub- $\mathrm{L}_{*}$ galaxies, hereafter low-mass galaxies, by using the hydrodynamics + N-body Adaptive Refinement Tree code (ART; Kravtsov et al. 1997; Kravtsov et al 2003) in the context of the $\Lambda$ CDM scenario (see also Colín et al. 2010).

In the recent literature, an unexpected observational trend for low-mass galaxies (stellar masses $M_{s}<310^{10} \mathrm{M}_{\odot}$ ) has been established: the lower $M_{s}$, the higher is on average the specific star formation rate $\left(\mathrm{sSFR}=\mathrm{SFR} / M_{s}\right)$. This trend, called "downsizing in sSFR", is seen at least out to $z \sim 1$ and it implies a delay in the $M_{s}$ assembling process. This delay should be larger for lower mass galaxies. The sSFR downsizing is difficult to be reproduced by $\Lambda$ CDM-based disk galaxy evolutionary models, which predict low values of sSFR for small galaxies at late epochs and, instead of downsizing, present a weak upsizing trend in sSFR (Firmani et al. 2010 and references therein). These models include SN-driven outflows, which are important for low-mass systems. In this paper we explore whether our simulated galaxies face such a difficulty.

\section{Numerical Setup}

Among the physical processes included in the hydrodynamics ART code are: metaldependent gas cooling, SF, thermal stellar feedback, self-consistent advection of metals, UV heating background source, etc. The cooling of gas takes into account atomic and 


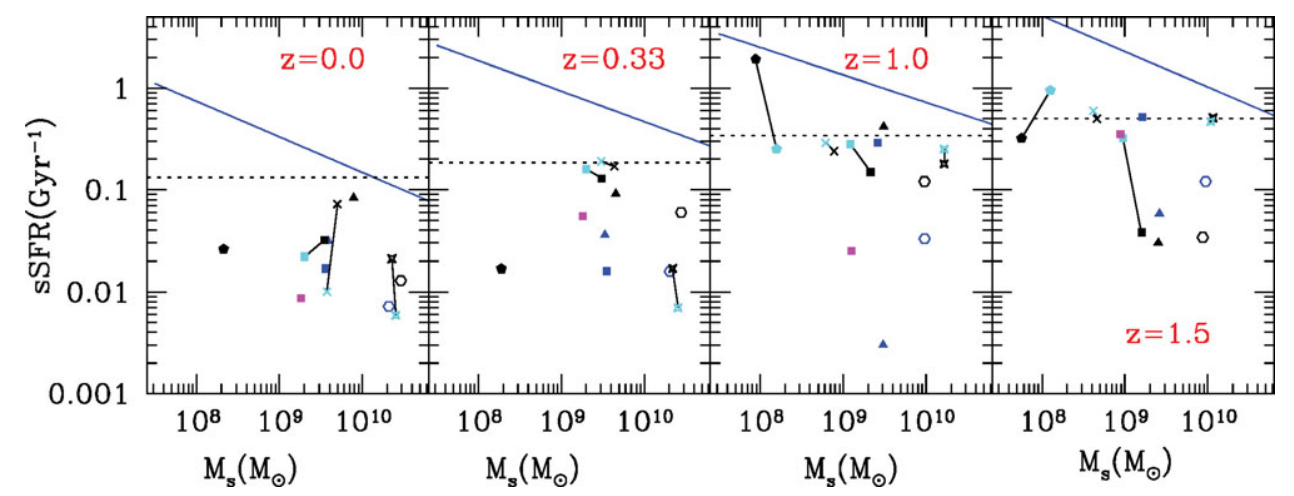

Figure 1. Specific star formation rate (sSFR) as a function of $M_{s}$, at four epochs, $z=0$, $0.33,1.0$, and, 1.5. Different simulated galaxies are denoted by different symbols while galaxies simulated with different resolution are connected by a line (color cyan represents the highest resolution). The symbols of galaxies simulated with different values of the parameters $f$ or $\Delta t$ (or resolution) have different color. The blue line at $z=0$ are data from Salim et al. (2007) (see their equation 12). The blue lines at other redshifts are the median values for different redshift bins taken from Santini et al. (2009): $0.3<z<0.6,0.6<z<1.0$, and $1.0<z<1.5$. Dashed lines show the values at each $z$ of $1 /\left[t_{H}(\mathrm{z})-1 \mathrm{Gyr}\right](1-R)$, where $R$ is the gas recycling factor, and it corresponds to galaxies with constant SFR.

molecular cooling. Star formation (SF) takes place in the coldest and densest collapsed regions, defined by $T<T_{\mathrm{SF}}$ and $\rho_{g}>\rho_{\mathrm{SF}}$, where $T$ and $\rho_{g}$ are the temperature and density of the gas, respectively, and $\rho_{\mathrm{SF}}$ is a density threshold. A stellar particle of mass $m_{*}=f m_{g a s}$ is placed in a grid cell, of mass $m_{g a s}$, where these conditions are simultaneously satisfied. The parameter $f<1$ is a measure of the local efficiency with which gas is transformed into stars. Although $n_{\mathrm{SF}}$, the hydrogen number density corresponding to $\rho_{\mathrm{SF}}$, is in principle a free parameter of the simulations, one can make an educated guess as to which values should be considered most realistic by noting that SF occurs almost exclusively in giant molecular clouds. Thus, it is natural to require that the density in a star-forming cell should be at least such that the cell's column density equals the threshold value for molecular Hydrogen and CO formation, $N \gtrsim 10^{21} \mathrm{~cm}^{-2}$ (Franco \& Cox 1986; van Dishoeck \& Black 1988). For a cell of 100-300 pc on a side, this translates into a $n_{\mathrm{SF}}=$ of a few $\mathrm{cm}^{-3}$. Here we set $n_{\mathrm{SF}}=6 \mathrm{~cm}^{-3}$ in all simulations (see Colín et al. 2010 for details).

In Table 1, we show with capital letters the simulated galaxies. Model galaxies with the same letter means same galaxy but simulated with different resolution or different values of the parameters $f$ or $\Delta t$ (see Table 1 for their definitions); for example, the difference between $\mathrm{C} 1$ or $\mathrm{C} 2$ is resolution, $\mathrm{C} 2$ is twice better resolved $†$. In second column, we show the total mass of the galaxy inside the virial radius $M_{T}=M_{D M}+M_{b a r}$, where $D M$ stands for dark matter. The mass of the $D M$ particle in the highest resolution zone is given in column fifth.

\section{Results}

Figure 1 shows the sSFR as a function of $M_{s}$ for all our simulations at different epochs. The SFR is the ratio between the stellar mass, produced during a certain time bin $d t$,

$\dagger$ Although the number of DM particles inside $R_{\mathrm{vir}}$ increases by about a factor of eight in these more resolved galaxies, the number of cells increases by much less because the refinement criteria (DM or gas mass in a cell) was fixed by the corresponding lower resolution simulations. 
Table 1. Parameter Simulations

\begin{tabular}{cccccc|cccccc}
\hline Galaxy & $\begin{array}{c}M_{T} \\
\left(M_{\odot}\right)\end{array}$ & $f^{1}$ & $\begin{array}{c}\text { Cooling }^{2} \\
\left(10^{6} \mathrm{yr}\right)\end{array}$ & $\begin{array}{c}m_{p} \\
\left(M_{\odot}\right)\end{array}$ & $\begin{array}{c}l_{\text {max }}^{3} \\
(\mathrm{pc})\end{array}$ & Galaxy & $\begin{array}{c}M_{T} \\
\left(M_{\odot}\right)\end{array}$ & $f^{1}$ & $\begin{array}{c}\text { Cooling }^{2} \\
\left(10^{6} \mathrm{yr}^{2}\right.\end{array}$ & $\begin{array}{c}m_{p} \\
\left(M_{\odot}\right)\end{array}$ & $\begin{array}{c}l_{\text {max }}^{3} \\
(\mathrm{pc})\end{array}$ \\
\hline $\mathrm{A} 1$ & $2.80 \times 10^{10}$ & 0.50 & 20.0 & $6.6 \times 10^{5}$ & 218 & $\mathrm{C} 2$ & $6.56 \times 10^{10}$ & 0.50 & 20.0 & $8.2 \times 10^{4}$ & 109 \\
$\mathrm{~A} 2$ & $2.48 \times 10^{10}$ & 0.50 & 20.0 & $8.2 \times 10^{4}$ & 109 & $\mathrm{D} 1$ & $9.52 \times 10^{10}$ & off & 40.0 & $7.5 \times 10^{5}$ & 218 \\
$\mathrm{~B} 1$ & $5.94 \times 10^{10}$ & 0.50 & 40.0 & $7.5 \times 10^{5}$ & 218 & $\mathrm{D} 2$ & $8.37 \times 10^{10}$ & 0.66 & 40.0 & $7.5 \times 10^{5}$ & 218 \\
$\mathrm{~B} 2$ & $5.31 \times 10^{10}$ & 0.50 & 40.0 & $9.4 \times 10^{4}$ & 109 & $\mathrm{E} 1$ & $3.04 \times 10^{11}$ & 0.50 & 40.0 & $7.5 \times 10^{5}$ & 218 \\
$\mathrm{~B} 3$ & $5.76 \times 10^{10}$ & 0.20 & 40.0 & $9.4 \times 10^{4}$ & 109 & $\mathrm{E} 2$ & $3.05 \times 10^{11}$ & 0.50 & 40.0 & $9.4 \times 10^{4}$ & 109 \\
$\mathrm{~B} 4$ & $5.11 \times 10^{10}$ & 0.50 & 10.0 & $9.4 \times 10^{4}$ & 109 & $\mathrm{~F} 1$ & $4.04 \times 10^{11}$ & off & 40.0 & $7.5 \times 10^{5}$ & 218 \\
$\mathrm{C} 1$ & $7.79 \times 10^{10}$ & 0.50 & 20.0 & $6.6 \times 10^{5}$ & 218 & $\mathrm{~F} 2$ & $3.58 \times 10^{11}$ & 0.66 & 40.0 & $7.5 \times 10^{5}$ & 218 \\
\hline
\end{tabular}

Notes:

${ }^{1}$ The parameter $f$ measures the fraction of gas mass, in a star forming cell, that is transformed into stars.

${ }^{2}$ The interval of time $\Delta t$ during which cooling is turned off after a SF event in order feedback may work.

${ }^{3}$ The cells in the finest grid measures $l_{\text {max }}$ on a side.
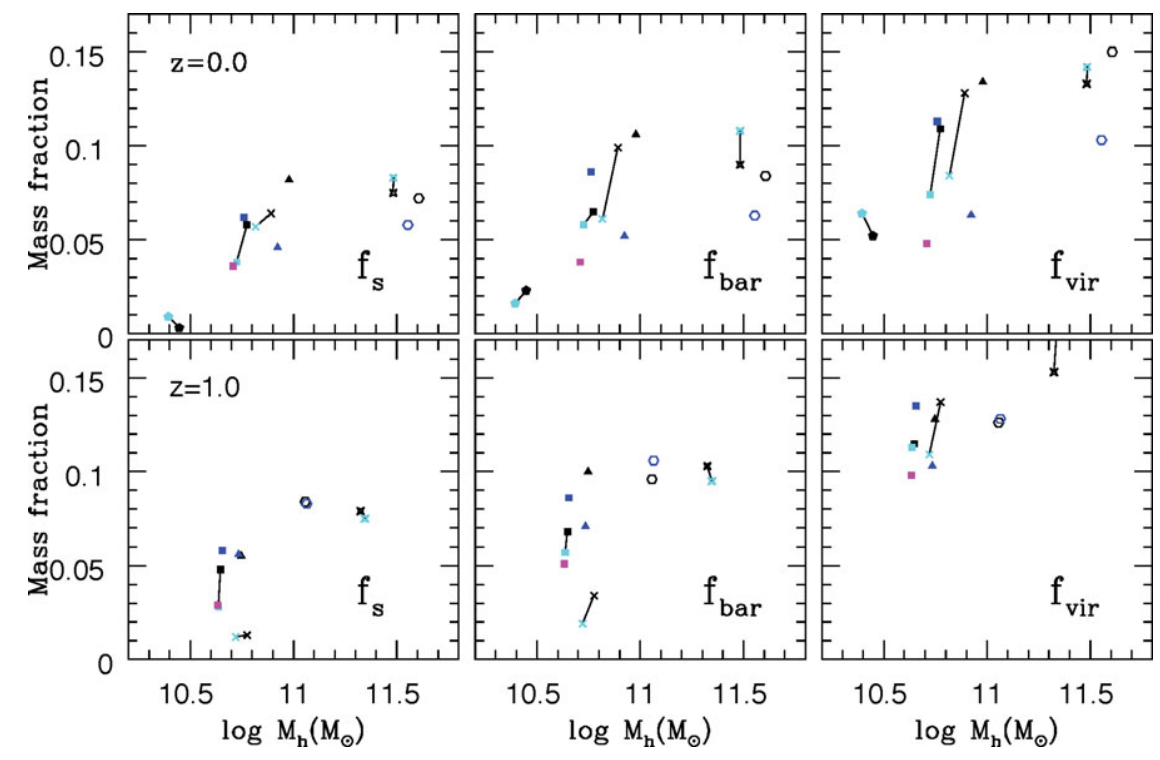

Figure 2. Mass fractions as a function of the mass of the halo, dark matter plus baryonic mass inside the virial radius, at two epochs, $z=0$ and 1 , for the different modeled galaxies, shown in Table 1. Galaxies are denoted by the same symbol and color code as in Figure 1. A trend can be clearly appreciated, the amount of baryons inside the galaxy, $5 R_{e}$ or $R_{\mathrm{vir}}$, decreases as the mass of the galaxy gets smaller.

taken here equal to $100 \mathrm{Myr}$, around $z$, and $d t$. The blue line at $z=0$ is a linear fit to star-forming galaxies (AGN excluded) from the SDSS (Salim et al. 2007); the scatter around this fit is large, $1 \sigma \approx 0.5$ dex. The blue lines in the other panels are the median values of observational inferences in Santini et al. (2009); from left to right, $0.3<z<0.6$, $0.6<z<1.0$, and $1.0<z<1.5$. These inferences are roughly representative of many more observational studies appeared in the last years (see more references therein and in Firmani et al. 2010). As in the observational samples, our galaxies have on average a sSFR that increases towards higher redshifts.

The same galaxy simulated with different (reasonable) sub-grid parameters may have different values of SSFR at a given $z$. We notice also that the SFR has an intrinsic fluctuating behavior (see Colín et al. 2010). The increase in resolution affects the sSFR, in most of the cases lowering even more the values of sSFR. Despite all these differences, 
our results show for all cases that the lower the mass, the smaller the sSFRs of the simulated galaxies with respect to observations out to $z \sim 1.5$.

Figure 2 shows the different mass fractions, stellar, $f_{s}=M_{s} / M_{T}$, baryon, $f_{\text {bar }}=\left(M_{s}+\right.$ $\left.M_{g}\right) / M_{T}$, and, virial, $f_{v i r}=M_{b a r} / M_{T}$, computed for the different simulated galaxies, shown in Table 1. Symbol and color code are as in Figure 1. The stellar fraction is computed inside five effective radii, $R_{e}$, defined as the half-mass stellar radius of the galaxy. The baryonic mass in $f_{\text {bar }}$ considers stars and gas at $T<10^{4} \mathrm{~K}$ inside $5 R_{e}$; $f_{v i r}$ uses all baryonic mass within $R_{\mathrm{vir}}$. In all three cases, $M_{T}$ is the total galaxy mass (see Table 1). At the two $z^{\prime}$ s a clear trend is seen: the amount of baryons decreases as $M_{T}$ decreases. This is clearly a result of the stellar thermal feedback: all of our lowmass simulated galaxies developed in greater or lesser extent gas blow-away episodes, with the strength of this galactic wind being a very sensitive function of potential well of the galaxy. This trend is in agreement with several direct and indirect observational estimates, though the values for $f_{s}$ or $f_{\text {bar }}$ (e.g. , Guo et al. 2010; Behroozi, Conroy \& Wechsler 2010), are at least a factor three lower than our results.

\section{Conclusions}

Our ART simulations are able to produce roughly realistic sub- $\mathrm{L}_{*}$ galaxies. The results depend on the values of the sub-grid parameters and resolution. However, for all the possibilities considered here, our results confirm that (i) $\Lambda$ CDM-based galaxies are not able to reproduce the observable phenomenon of downsizing in sSFR, and (ii) their stellar mass fractions, in spite of the feedback-driven outflows, are too high with respect to observational inferences. According to observations, low-mass galaxies have SFRs much higher than their past average, even at $z \sim 1$; dashed lines in Fig. 1 show the case if one assumes a constant SFR: galaxies above (below) these lines are forming stars at a higher (lower) rate than the past average. If the observational inferences related to the downsizing in sSFR are definitively confirmed, then in order to reproduce them, galaxies formed in low-mass $\Lambda$ CDM halos should delay their active SF phase towards later epoch. This delay should be larger for lower mass galaxies. It is not clear how to attain this in models and simulations; new astrophysical ingredients are probably necessary. For high masses, the AGN feedback is commonly invoked to explain mass downsizing. For lowmass galaxies this mechanism does not apply because observations show that the AGN phenomenon is not common in these galaxies.

\section{References}

Colín, P., Avila-Reese, V., Vázquez-Semadeni, E., Valenzuela, O., \& Ceverino, D. 2010, ApJ, 713,535

Behroozi, P. S., Conroy, C., \& Wechsler, R. H. 2010, ApJ, 717, 379

Firmani, C., Avila-Reese, V., \& Rodríguez-Puebla, A. 2010, MNRAS, 404, 1100

Franco, J., \& Cox, D. P. 1986, PASP, 98, 1076

Guo, Q, White, S., Li, Ch., \& Boylan-Kolchin, M. 2010, MNRAS, 404, 1100

Kravtsov, A. V., Klypin, A. A., \& Khokhlov, A.M., 1997, ApJS, 111, 73

Kravtsov, A. V. 2003, ApJ (Letters), 590, 1

Salim, S. et al. 2007, ApJS, 173, 267

Santini P. et al., 2009, A\&A, 504, 751

van Dishoeck, E. F., \& Black, J. H. 1988, ApJ, 334, 771 